\title{
The FFT Harmonic Analysis in Frequency Converters based on Wavelet Denoising
}

\author{
Shuxi Liü, a, Shan $\mathrm{Li}^{2, b}$ and Xiaoduo Yang ${ }^{3, \mathrm{c}}$ \\ ${ }^{1}$ Chongqing University of Technology, Chongqing, China \\ ${ }^{2}$ Chongqing University of Technology, Chongqing, China \\ ${ }^{3}$ Chongqing University of Technology, Chongqing, China \\ ashuxi@cqut.edu.cn, blishan@cqut.edu.cn, c154154816@qq.com
}

\begin{abstract}
Keywords: Harmonic analysis, frequency converter, wavelet, denoising.
Abstract. Running converters causes harmonics in the electrical system, so it is critical to detect and analyze the harmonics in the power signals. However, the various noises could heavily jeopardize the accuracy of detection and analysis of harmonics in the subject. In this paper, wavelet denoising is applied in the FFT harmonic analysis to reduce the negative effects of both power grid and measurement noises. This method uses Stein's unbiased risk estimation (SURE) to acquire threshold and then soft-threshold the high frequency coefficients of the output signals of the converter, which drives a three-phase asynchronous motor. After we reconstruct the wavelet coefficients, the denoising-handled output signals of converter are processed with the FFT analysis in Matlab. The results show that the wavelet denoising has a positive effect on the FFT analysis and the harmonics of the output become more explicit with the increase of the motor speed.
\end{abstract}

\section{Introduction}

The high performance frequency converters have caused fundamental change in the field of electric drive control around the world. This technology is a very combination of computer control, intelligent control, optoelectronic technology, power electronics and even cooling technology. However, with the wide use of power electronic devices in frequency converters, the accompanying harmonic has a heavy influence on the quality of the power grid, which would pose a threat to the safe and stable operation of the power grid. Therefore, it is important to detect and analyze the harmonics of the output signals of the frequency converters and conclude the cause and changing laws of the harmonics of the frequency converters. Meanwhile, there are kinds of noises existed in the signals during the detection of harmonics. Adding signals denoising process in the harmonics analysis is a necessary method to improve the accuracy of harmonics analysis in frequency output signals ${ }^{[1-3]}$.

In this paper, a three-phase asynchronous motor driven by an AC-DC-AC Converter, which the rectifier side is diode uncontrollable rectifier, is applied in the harmonics analysis. Firstly, we acquire the output currents and voltages of the frequency converter at three different speed $(500 \mathrm{r} / \mathrm{min}$, $800 \mathrm{r} / \mathrm{min}$ and $1200 \mathrm{r} / \mathrm{min}$ ) of the three-phase asynchronous motor. Then, we use the wavelet denoising method on the signals with noises and run FFT analysis on the denoised signals to get frequency, amplitude of each harmonic. Both these two steps are accomplished on the Matlab software. The results show that the adding wavelet denoising process before FFT analysis reduces the negative influence on the accuracy of harmonics detection. Moreover, the fifth and seventh harmonics are the main harmonics in the output voltage and current signals of the frequency converter, and with the increase of the speed, the percentage of harmonics drops but still exists.

\section{Principle of wavelet denoising}

Wavelet denoising is a major application in the wavelet analysis. There are three main steps of wavelet denoising process. The first one is wavelet multi-scale decomposition. Hence the output current and voltage signals obtained by oscilloscope are a series of discrete data, this step is based on the DWT (Discrete wavelet transform) (1): 


$$
C_{j, k}(t)=\int_{-\infty}^{\infty} f(t) \psi_{j, k}^{*}(t) d t=\left\langle f, \psi_{j, k}\right\rangle
$$

The $f(t)$ refers to the signal while the $\psi_{j, k}$ is the suited wavelet basis function. After selecting the specific wavelet basis function, we can get the coefficients of the next several levels by doing DWT on the original signal. Fig. 1 shows the three scales of decomposition of a signal, where the $\mathrm{cAx}(\mathrm{x}=1$, $2,3)$ are the low-frequency coefficients of every level while the $\mathrm{cDx}(\mathrm{x}=1,2,3)$ are the high-frequency of every level in the decomposition ${ }^{[4,5]}$.

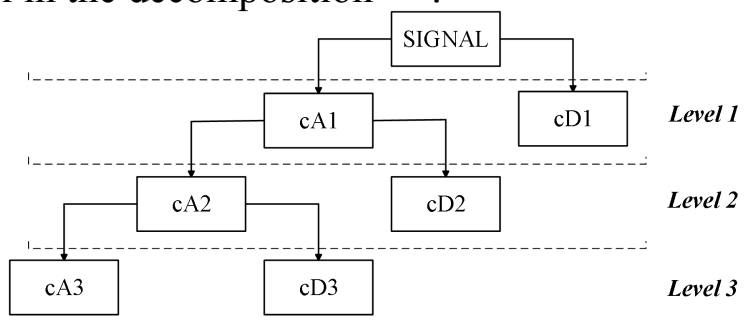

Fig. 1 Decomposition in three scales

The second step of wavelet denoising process is using the optimized threshold to handle the high-frequency coefficients with soft-thresholding method at every decomposition level obtained in step one method. Considering that the output current and voltage signals of frequency converter are used for harmonic analysis, simply eliminating the high frequency part would loss the details of the useful signals. The Stein's unbiased risk estimation (SURE) threshold rule has the ability to extract the weak signals in high frequency parts ${ }^{[6-7]}$, therefore, the soft-threshold calculation based on SURE rule is the main content of this step. Firstly, calculate the noise intensity through equation (2):

$$
\sigma=\frac{1}{0.6745} \sum_{i=0}^{N-1}\left|C_{i}^{k}\right|
$$

Where $C_{i}^{k}$ is coefficients in the level $\mathrm{k}$ while $\mathrm{N}$ is the decomposition levels. Then, the general threshold $T_{1}$ is calculated by (3):

$$
T_{1}=\sigma \sqrt{2 \ln N}
$$

Lastly, rank the square of coefficients at any level from small to large in $W=\left[w_{1}, w_{2}, \ldots, w_{N}\right]$ and get the estimation vector $E=\left[e_{1}, e_{2}, \ldots, e_{N}\right]$ through $^{[8]}(4)$ :

$$
e_{i}=\frac{\sum_{k}^{i} w_{k}+n+(n-1) w_{i}-2 i}{N}
$$

SURE thresholds $T_{2}$ are obtained by equation (5):

$$
T_{2}=\sigma \sqrt{w_{b}}
$$

Where the $w_{b}$ is the corresponding $\mathrm{b}$ in the $e_{b}$ when we use the minimal value $e_{b}$ as our estimation. Once we get the general thresholds and SURE thresholds, the final thresholds $T_{3}$ are chosen by (6):

$$
T_{3}=\left\{\begin{array}{l}
T_{1}, \eta>\mu \\
\min \left(T_{1}, T_{2}\right), \eta \leq \mu
\end{array}\right.
$$

In the above-mentioned formula, the $\eta=\left(S_{w}-N\right) / N$ while the $\mu=\left(\log _{2} N\right)^{3 / 2} N^{1 / 2}$ and the $S_{w}=\sum_{i=1}^{N} w_{i}^{2}$. The final threshold $T_{3}$ is the very threshold that we use to soft threshold the coefficients in every decomposition level acquired in step one ${ }^{[9,10]}$.

The third step of denoising process is based on the wavelet inverse transformation. The new coefficients, which are handled in soft-thresholding in step two, are implemented with wavelet reconstruction through the wavelet inverse transformation in order to get the denoised signals. A noised sine wave is experienced with the proposed wavelet denoising method and the results are shown in Fig.2. 


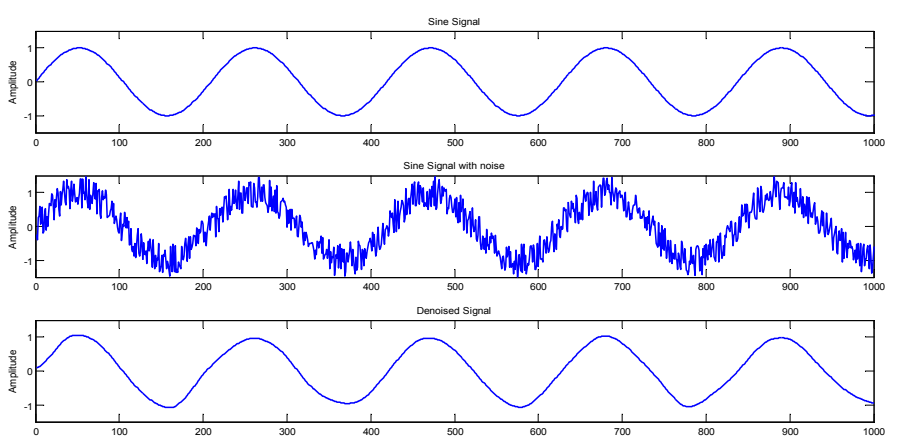

Fig. 2 Effect of the proposed wavelet denoising method

After this step, the denoised signals are planned for FFT to analyze the harmonics in the frequency converters.

\section{Harmonics analysis}

In this paper, a three-phase asynchronous motor driven by an AC-DC-AC Converter is applied in the experiment. In order to find out the harmonic and its changes in different converters' working status, we tested the output currents and voltages when the converter drove a three-phase asynchronous motor under three conditions $(500 \mathrm{r} / \mathrm{m}, 800 \mathrm{r} / \mathrm{m}$ and $1200 \mathrm{r} / \mathrm{m})$.

\section{(1) Wavelet denoising process}

Wavelet denoising process used the method produced in the 2 nd section. After the converter steadily drove the three-phase asynchronous motor, the current and voltage signals on phase A were captured by an oscilloscope. For example, Fig. 3 shows the output signals of phase A when the motor was at $1200 \mathrm{r} / \mathrm{m}$ speed, where the red line represents the current while the blue line refers to the output voltage signal. From Fig.3 we can know that the voltage waveform is a series of square wave with massive glitches and the inductive load made the output current approximate sine wave. The waveforms at the speeds of $500 \mathrm{r} / \mathrm{m}$ and $800 \mathrm{r} / \mathrm{m}$ are omitted because they are similar to the $1200 \mathrm{r} / \mathrm{m}$ condition.

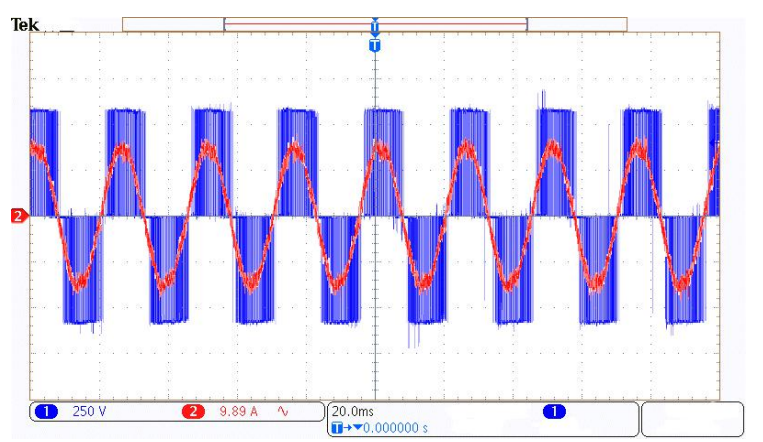

Fig. 3 Output signals at $1200 \mathrm{r} / \mathrm{m}$ speed
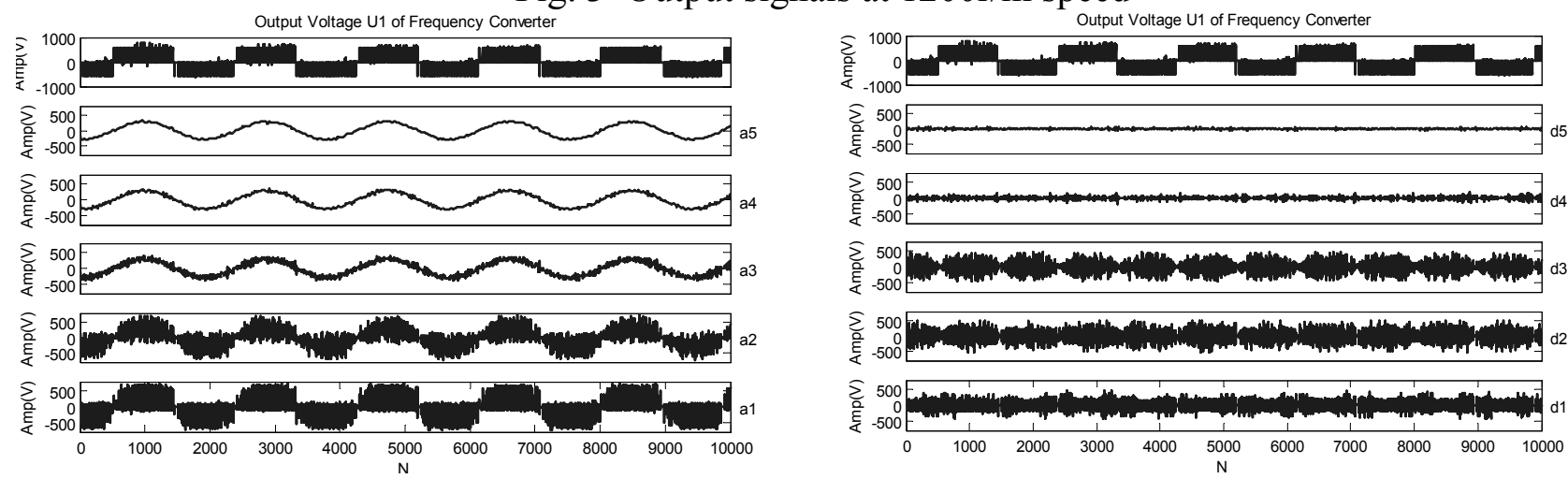

Fig. 4(a) Coefficients of the wavelet decomposition

Fig. 4(b) Coefficients of the wavelet decomposition

After captured from the oscilloscope, the current and voltage signals were processed wavelet decomposition through Matlab software. Considering that the sampling frequency of oscilloscope is 
$5 \mathrm{kHz}$, the signals were decomposed in 5 levels and the db10 wavelet function was used in the transform. Fig.4(a) and Fig.4(b) show both the approximation and detail coefficients of the voltage signal at $1200 \mathrm{r} / \mathrm{m}$ condition after wavelet decomposition.

According to the SURE threshold selection rule and wavelet denoising method in the 2nd section, the decomposed 5 levels' coefficients were used to calculate the final threshold $T_{3}$ and then threshold the coefficients with $T_{3}$. Later, we reconstructed the signals with the new coefficients in order to finish the denoising. Fig.5 demonstrates the outcomes of wavelet denoising, the noise has been largely removed.
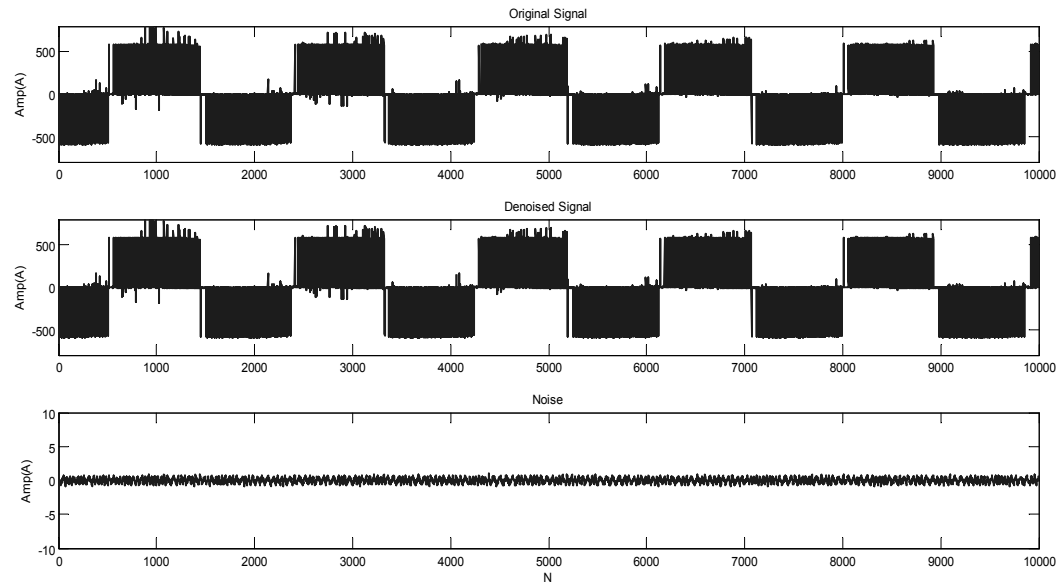

Fig. 5 Results of denoising in voltage

\section{(2) FFT analysis}

The wavelet denoising process is just the first step to analyze the harmonics of the output signals of the frequency converter. After processed the wavelet denoised process, the output current and voltage would run fast Fourier transform to find out the exact categories and percentages of the harmonics that affect the motor.
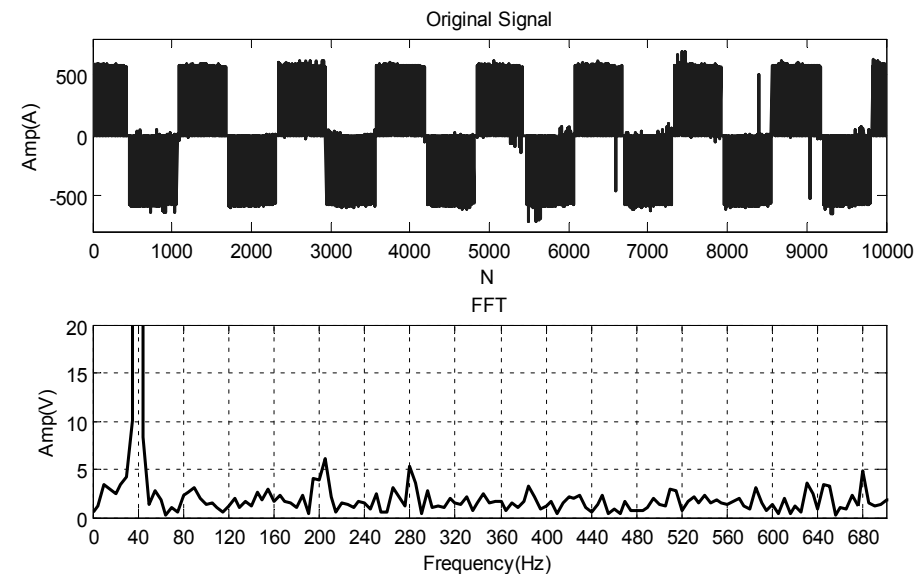

Fig. 6 FFT analysis of the output voltage at 1200r/m speed

Fig.6. illustrates the harmonics of current and voltage of output at the speed of $1200 \mathrm{r} / \mathrm{m}$, which the fundamental frequency is $40 \mathrm{~Hz}$. The results tell us that the voltage harmonics that generated by frequency converter mostly concentrate in the 5 th, 7 th and above 17 th harmonics while the harmonics in current mainly exist in the $3 \mathrm{rd}, 5$ th and 7 th.

Table 1 gives the exact percentages of typical harmonics at three different speeds $(500 \mathrm{r} / \mathrm{m}, 800 \mathrm{r} / \mathrm{m}$ and $1200 \mathrm{r} / \mathrm{m}$ ) after we tested and running FFT analysis. From the table 1, we can figure out that the low-order harmonics (especially the 5th, 7th harmonics) exist in the operation of frequency converter when it drives three phase asynchronous motor. Furthermore, the harmonics rises with the increase of the motor speed. 
Table 1 Percentages of typical harmonics at 3 kinds of speeds

\begin{tabular}{rrlllllll}
\hline & 5 th & 7 th & 9 th & 11 th & 13 th & 15 th & 17 th & 19 th \\
\hline $500 \mathrm{r} / \mathrm{m}$ & $1.59 \%$ & $1.28 \%$ & $0.25 \%$ & $0.07 \%$ & $0.09 \%$ & $0.10 \%$ & $1.41 \%$ & $0.93 \%$ \\
$800 \mathrm{r} / \mathrm{m}$ & $2.21 \%$ & $1.44 \%$ & $0.33 \%$ & $0.11 \%$ & $0.06 \%$ & $0.15 \%$ & $1.98 \%$ & $1.66 \%$ \\
$1200 \mathrm{r} / \mathrm{m}$ & $2.40 \%$ & $2.08 \%$ & $0.42 \%$ & $0.09 \%$ & $0.10 \%$ & $0.23 \%$ & $2.03 \%$ & $1.58 \%$ \\
\hline
\end{tabular}

\section{Conclusions}

In this paper, a wavelet denoising process is applied in the harmonics analysis of frequency converters to remove the noises caused by power grid and measurement. The results show us that the wavelet denoising method based on SURE threshold selection has a positive effect on the noises removing and the denoised signals which are analyzed through FFT give us a better outcome of the harmonics analysis. Today, with the massive operation in frequency converters, frequency converters cause great impact in power grid while operating, we should have more effective ways of detecting and analyze the harmonics.

\section{References}

[1] Li Dongpo. Study on the effect of frequency converter on the performance of three phase asynchronous motor, Industrial Engineering and technology, 2013(08):39.(In Chinese)

[2] Xiu, L. Digital-to-Frequency Converter: A Component Enabling New Application Fields in From Frequency to Time-Average-Frequency: A Paradigm Shift in the Design of Electronic System , Vol. 1, Wiley-IEEE Press, 2015, pp.180

[3] Badrzadeh, B., Gupta, M., Singh, N., Petersson, A., Max, L., Hogdahl, M. Power system harmonic analysis in wind power plants - Part I: Study methodology and techniques, in Industry Applications Society Annual Meeting (IAS), 2012 IEEE, pp.1-11, Oct. 2012

[4] Julio Barros and Ramón I. Diego. Analysis of Harmonics in Power Systems Using the Wavelet-Packet Transform, IEEE Trans. On Instrumentation and Measurement, vol. 57, No. 1, Jan. 2008, pp. 63-69.

[5] G. S. Kumar, D. V. S. S. S. Sarma and C. Venkatesh. Wavelet transform based harmonic analysis and real power measurement, Power Electronics (IICPE), 2010 India International Conference on, New Delhi, 2011, pp. 1-6.

[6] Shuangbo Fei, Ruizheng Zhao. Image Denoising with Wavelet Threshold Based on SURE Rule, Journal of Beijing Jiaotong University, 2007(02):39. (In Chinese)

[7] Tianshu Qu, Yisong Dai, Shuqin Wang. Adaptive Wavelet Thresholding Denoising method based on Sure Estimation, Acta Electronica Sinica, 2002(02):2. (In Chinese)

[8] M. L. Hilton and R. T. Ogden. Data Analytic Wavelet Threshold Selection in 2-D Signal Denoising, IEEE Trans on Signal Processing, vol. 45, No. 2, 1997, pp. 496-500.

[9] Donghong Liu, Yongshun Zhang, Qainjun Ding. Wavelet Denoising based on SURE, Journal of Projectiles, Rockets, Missiles and Guidance, vol. 24, No. 1, 2004, pp. 356-359.

[10] Shuwen Dang, Weifeng Tian, Zhihua Jin. De-Noising Stochastic Noise in FOG Based on Second-Generation DB4 Wavelet and SURE-Threshold, Journal of Wuhan University English Edition, vol. 6, 2009, pp. 494-498. 